\title{
Self-Localization in Wireless Sensor Networks Using Particle Filtering with Progressive Correction
}

\author{
Thomas Hanselmann*, Yu Zhang ${ }^{\dagger}$, Mark Morelande*, Mohd Ifran Md Nor*, Jonathan Wei Jen Tan*, \\ Xing-She Zhou ${ }^{\dagger}$, and Yee Wei Law* \\ *Dept. EEE, The University of Melbourne, Parkville, VIC 3010, Australia. Email: t.hanselmann@ee.unimelb.edu.au \\ ${ }^{\dagger}$ School of Computer Science, Northwestern Polytechnical University, Xi’an, 710072, China. \\ Email: \{zhangyu, zhouxs $\} @$ nwpu.edu.cn
}

\begin{abstract}
A centralized self-localization algorithm is used to estimate sensor locations. From the known positions of at least 3 anchor nodes the remaining sensor positions are estimated using an efficient particle filter (PF) with progressive correction. The measurement model is a simple two-parameter log-normal shadowing model, where the parameters are estimated concurrently. Experiments using Crossbow Imote2 motes show that an error of less than $16 \%$ is achievable in an indoor environment. The results demonstrate that by using $\mathrm{PF}$ with progressive correction, a small number of measurements and a simple signal propagation model are sufficient to give low localization errors.
\end{abstract}

\section{INTRODUCTION}

Wireless sensor networks (WSNs) have become more and more accessible in many domains. They can be used to collect sensor data. However, sensor data often only make sense if the locations of the sensors are known. With mobile and selfforming adhoc networks, localization of individual nodes is often crucial to further signal processing.

While some of the sensor nodes' positions maybe known, e.g. via GPS localization, or they may be fixed at known positions, the remaining sensors have to be localized based on some measurement model that links those measurements to distances between the nodes.

Our algorithm uses the received signal strength (RSS), as the values are readily available in commercial radio chips and can be piggy-backed on existing communication links at no extra cost. Unfortunately RSS is often unreliable and extremely noisy. In the Imote 2, only an 8-bit RSS value is available, which can lead to strong quantization noise. An extensive work has been done by Lymberopoulos et al. [7] to empirically determine RSS variability, using 1.1 inch wire antennas that are optimally matched. Complementary to their research, we experimented with the onboard chip-antenna, and an external antenna.

In this paper, position estimation is formulated as a 2-D localization problem, however, it is easy to extend the state space to estimate full 3-D positions. More problematic may be the antenna orientation in 3-D as observed by [7]. However, as we use standard $2.4 \mathrm{GHz}$ wireless router antennas of a larger (external) $\operatorname{size}^{1}$, the effect may not be as drastic as with the

This work is partly supported by National Natural Science Foundation of China under Grant No.60573161 and National Key Technology R\&D Program of China under Grant No.2007BAD79B02.

${ }^{1}$ The effective internal antenna size is unknown. small 1.1 inch wire antennas. The main goal is to have the effective internal antenna as obstruction-free as possible so that the RSS values fit the log-normal shadowing model as well as possible.

Algorithms for sensor networks should be decentralized, low-complexity in computation, efficient in the use of measurements and scalable in the number of nodes:

Decentralization While currently the proposed algorithm is centralized, it is applicable to larger networks. Larger networks can be divided into smaller sub-networks which have some overlap (at least 3 anchor nodes). In all the sub-networks with at least three anchor nodes of known position, the proposed algorithm can be used.

Low complexity The requirement for low complexity is addressed by using only RSS measurements and the application of a simple two-parameter log-normal shadowing model.

Efficiency The efficiency requirement is addressed by formulating the problem as a joint Bayesian estimation problem for all the localization parameters, which is solved by an efficient PF with progressive correction. Progressive correction is a multi-stage procedure in which samples are obtained from a series of intermediate distributions which approach the posterior distribution typically in just a few iterations [12].

Scalability Scalability is achieved by using measurements only between neighboring pairs of sensor nodes. Naturally, large sensor networks are sparse and multi-hop networks otherwise sensing resources are wasted. An efficient algorithm for localization in distributed sensor networks based on radio interferometric positioning system (RIPS) measurements has been proposed by Brazil et al. [2]. The efficient use of measurements is of great importance and has not been investigated extensively. Many papers show only simulations with measurements based on Gaussian noise assumption and the assumption that measurements are cost-free and available in vast numbers. This paper shows that a small number of measurements, even with extreme outliers according to the simple measurement model used, is sufficient to achieve a reasonable accuracy. Even with 6 available sensor nodes we do not need all 30 possible pair measurements to achieve fairly accurate position estimates, which is very promising for tracking mobile nodes or adapting to changing environmental conditions.

In short, our contribution is an efficient and accurate 
localization algorithm based on the Bayesian estimation of two radio model parameters, given only a few measurements.

This paper is organized as follows. Section II introduces some related work. Section III describes our measurement model. Section IV characterizes the antenna used for this work. Section V gives the detail of the algorithm. Section VI gives some experimental results. Finally, Section VII concludes.

\section{RELATED WORK}

Localization schemes for WSNs can be divided into rangebased and range-free schemes. Range-based methods use range measurements (e.g., RSS, time of arrival etc.) while range-free techniques use other types of information such as connectivity. There is a vast amount of literature on localization (refer to [8] for a comprehensive review). Here, we only briefly discuss some relevant range-based techniques that also employ PFs. The need to use PF is best summarized in [13], which states that Extended Kalman Filter (EKF) "fails to provide an acceptable performance especially when the number of quantization levels is small".

An extensive comparative study done by Elnahrawy et al. [4], range-based localization algorithms are classified into two groups: area-based and point-based. An area-based algorithm gives some kind of confidence that the true location is within the designated area. They argue that the key property of area-based algorithms is that they can trade accuracy (likelihood to be in area) for precision (size of area). A pointbased algorithm only gives a location without any measure of (un)certainty. The algorithm proposed here is an area-based algorithm, where the particle spread for a sensor location represents the confidence, or more precisely, the probability distribution of the (multi) sensor location(s).

Historically, PF (also called the sequential Monte Carlo method) is used in robotics to locate mobile robots. Perhaps the earliest known use of PF for WSN localization is by $\mathrm{Hu}$ et al. [5]. Dil et al. [3] improve the accuracy by using range information from anchors that are one/two hops away from an unlocalized node, at the expense of communication complexity. A PF is also used in [10] but for the proposed technique to work, an extensive off-line calibration exercise, involving the placement of each node at multiple locations, has to be undertaken. Another PF-based work [6] does not prescribe any particular signal propagation model, but the results were derived from simulations. Our results are based on actual measurements. Baggio et al. [1] introduce a 'boxing' method for reducing the number of samples that need to be drawn. By using progressive correction, our algorithm also only needs a small number of samples.

Compared to existing work that use multiple range measurements (e.g., [11] use time of arrival and direction of arrival), our scheme has minimal hardware requirements, i.e., only the ability to measure RSS. While Patwari et al. [14] also use a simple model and only RSS, their maximum likelihood estimation method is more sensitive to fading outliers.

In this paper, the localization of 2-D positions of $M$ unknown nodes is treated as a non-linear parameter estimation problem in a Bayesian framework with PFs. The framework is presented in detail in [9] for RIPS measurements, but it can be used with any suitable measurement model for other measurements as well. For completeness, a summary of the framework is given in Section V.

\section{MODELING}

Given $S$ sensor nodes at locations $\mathbf{x}_{i}=\left[x_{1}, x_{2}\right]^{T}, i=$ $1, \ldots, S$, with $K$ known locations $(i=1, \ldots, K)$ and $M=$ $S-K$ unknown locations $(i=K+1, \ldots, S)$, the goal is to estimate these $2 M$ parameters. Let $\mathrm{x}$ be the concatenation of all $\mathbf{x}_{i}$. In Bayesian modeling, a prior distribution $p(X)$ on a state $X$ (here, the $2 M$ parameters) is used and updated using the model likelihood to achieve a posterior distribution $p(X \mid D)$ that accounts for the measurements $D$. State estimates $\hat{X}$ may be obtained by calculating the first moment $E[X]$ or the location of maximum posterior probability $\hat{X}=\operatorname{argmax} p(X)$.

\section{A. Measurement Model}

The measurement model is based on the simple freespace expansion of a radial wave from a point source with transmission power $P_{0}$, located at $\mathbf{r}_{0}$. The power density on a ball located at $\mathbf{r}_{0}$ of radius $R$ is then $p_{A}(R)=\frac{P_{0}}{4 \pi^{2} R^{2}}$. At the receiver's side, at distance $R$ from the transmitter, a small antenna area $d A$ will receive a transmitted power of $P(R)=p_{A}\left(\mathbf{r}_{0}, R\right) d A$ which will be proportional to the send power $P_{0}$ and to $\frac{1}{R^{2}}$. To account for atmospheric transmission loss, antenna loss, symbol detection loss etc., it is convenient to modify this inverse power law as follows

$$
P(R)=P_{0}\left(\frac{R_{0}}{R}\right)^{\alpha}
$$

where $\alpha$ accounts for the losses and $R_{0}$ is a minimal distance that spherical antenna model from a point source applies with a real antenna. It is convenient to measure powers in decibels, which suits the hardware implementation as the RSS is given as an 8bit value in $\mathrm{dBm}$. Hence, the measurement model is:

$$
\begin{aligned}
P_{d B m}(R) & =10 \log _{10}\left(\frac{P(R)}{1 m W}\right) \\
& =P_{0, d B m}-10 \alpha \log _{10}\left(\frac{R}{R_{0}}\right)
\end{aligned}
$$

with $R_{0}=1 \mathrm{~m}$. This is often referred to as the log-normal shadowing model [14].

\section{Antenna Characterization}

To find out whether the above model is suitable for practical real-world modeling, two experiments - one with the Imote 2's onboard chip antenna and one with an external monopole antenna - were performed to measure the antenna characteristics. 12 different directions and up to 10 different radial distances between $1 \mathrm{~m}$ and $10 \mathrm{~m}$ were used.

\section{A. Onboard Chip Antenna}

To measure the antenna profile, a receiver was placed in the center of a laboratory room. The receiver was powered 
by a USB cable instead of a battery pack because a battery pack causes considerably more interference. A transmitter in the form of a battery-powered Imote 2 with its antenna facing the receiving Imote 2, was placed at different radii and angles. Figures 1-2 show the received powers and Figure 3 shows the directional radial profile at the 12 directions. The black line indicates a least-square fit of the log-shadow model.

\section{B. External Monopol Antenna}

To measure the antenna profile, a receiver was placed at the center of a laboratory room. As before, the receiver was powered by a USB cable; and a transmitter in the form of a battery-powered Imote 2 with its antenna facing the receiving Imote 2, was placed at different radii and angles. Figures 4-5 show the received powers and Figure 6 shows the directional radial profile at the 12 directions. The black line indicates a least-square fit of the log-shadow model. Compared to the onboard chip antenna, the radial profile of the external antenna is far more consistent and the estimated parameters for $P_{0}=$ $-3.91 \mathrm{dBm}$ and $\alpha=2.91$ seem to be quite reasonable.

\section{BAYESIAN NODE LOCALIZATION}

In a Bayesian framework, the goal is to minimize the expected squared error with respect to the posterior distribution. As the posterior distribution and hence the estimate of posterior mean cannot be found exactly, numerical approximations are used. A general-purpose method for non-linear problems is PF. Particles are drawn from a prior distribution or if not available an importance density to cover the possible parameter space. Bayesian update to calculate the posterior is done by multiplying the prior with the likelihood function which is normally obtained from the measurement model. With narrow likelihood functions, PFs suffer in practice from particle extinction (also termed particle collapse), especially in high-dimensional estimation problems. In this problem, there are $2 M$ position parameters to be estimated and possibly more model parameters. For larger $M$, this quickly turns into a high-dimensional problem; and strong non-linearity in the measurement model makes it an even harder problem, especially with limited computational resources available. While sampling from any importance density, covering the domain of interest of the posterior, the true posterior mean can be obtained in the limit of using an infinite number of particles, the goals here must be to obtain a good approximation with a small number of particles and to avoid particle extinction.

Therefore, the idea of progressive correction is used. This method iterates over several stages, starting with a diffuse likelihood function to get intermediate posterior distributions. The intermediate posterior distributions become progressively closer to the true posterior distribution. This is achieved by constraining the sequence of likelihoods to become closer, and at the last stage to be equal to the true model likelihood. Starting with diffuse likelihoods in early stages helps to prevent extinction of most of the particles at early stages. A standard PF would either be very inefficient or divergent. Similarly, a Bayesian framework with a simple Kalman Filter, or an EKF, would not be able to cope with the highly nonlinear model and the amount of noise from real hardware measurements.

Let $\mathbf{x}$ be the concatenation of known and unknown position parameters and $\mu(j, \mathbf{x})$ a slicing operator to extract the known or estimated position parameters of the $j$ th node. Let $\mathbf{d}$ be the vector of measurements with indicator function $\nu(k)=[i, j]$ such that the $t$ th element in $\mathbf{d}$ corresponds to $d_{i j}$ which is the RSS in $\mathrm{dBm}$ from node $i$ to node $j$. Let $l_{s}(\mathbf{d} \mid \mathbf{x})=l(\mathbf{d} \mid \mathbf{x})^{\Gamma_{s}}$ be the likelihood function at stage $s$, where $\Gamma_{s}=\sum_{j=1}^{s} \gamma_{j}$, $\gamma_{j} \in(0,1]$ and $\Gamma_{S}=1$. The true likelihood function, assuming independent measurements, is

$$
\begin{aligned}
l(\mathbf{d} \mid \mathbf{x}) & \left.\left.=\prod_{t=1}^{T} \mathcal{N}\left(d_{t}\right) \mid h\left(R_{t}\right) ; \sigma_{d}^{2}\right)\right) \\
R_{t} & =\|\mu(\nu(t)(1), \mathbf{x})-\mu(\nu(t)(2), \mathbf{x})\|
\end{aligned}
$$

where $h$ is the measurement model 2 with $\nu(t)(1)$ and $\nu(t)(2)$ being source and destination node index. $d_{t}$ is the $t$ th measurement and measurement noise is assumed to be additive Gaussian with variance $\sigma_{d}^{2}$.

Let $\pi_{s}(\mathbf{x}) \propto l_{s}(\mathbf{d} \mid \mathbf{x}) \pi_{0}(\mathbf{x}), s=1, \ldots, S$ be the posterior pdf according to the $s$ th likelihood, then

$$
\pi_{s}(\mathbf{x}) \approx C l(\mathbf{d} \mid \mathbf{x})^{\gamma_{s}} \sum_{i=1}^{n} w^{s-1, i} g_{s-1}\left(\mathbf{x}-\mathbf{x}^{s-1, i}\right)
$$

where $C$ is a normalizing constant, $g_{s-1}$ is a kernel density and the summation is the approximation of $\pi_{s-1} \cdot \pi_{s}(\mathbf{x})$ can be interpreted as an $n$-mixture with $i$ as an index to the $i$ th mixture component $C l(\mathbf{d} \mid \mathbf{x})^{\gamma_{s}} w^{s-1, i} g_{s-1}\left(\mathbf{x}-\mathbf{x}^{s-1, i}\right)$. Sampling from (5) may then be seen as drawing a mixture index and a parameter sample from the corresponding mixture component. Here, only the blind progressive correction is described; for more details see [9]. Thus sampling is done from the proposal density $q_{s}=\pi_{s-1}$ and the algorithm is summarized in Table I. The kernel density functions $g_{s-1}$ have been chosen as zero-mean Gaussians with covariance matrix $\Sigma_{s-1}$ appropriately chosen (basically a weighted covariance matrix from the particle samples, see [15, Chapter 4]).

1) Select $\gamma_{1}, \ldots, \gamma_{S}$.

2) For $i=1, \ldots, n$, draw $\mathbf{x}^{0, i} \sim \pi_{0}$ and set $w^{0, i}=$ $1 / n$.

3) For $s=1, \ldots, S$ :

a) Resample: Select indices $j^{s, 1}, \ldots, j^{s, n}$ such that $P\left(j^{s, i}=k\right)=w^{s, k}$.

b) For $i=1, \ldots, n$, draw $\mathbf{x}^{s, i} \sim g_{s-1}\left(.-\mathbf{x}^{s-1, j^{s, i}}\right)$.

c) For $i=1, \ldots, n$, compute weights $w^{s, i}=$ $C l\left(\mathbf{d} \mid \mathbf{x}^{s, i}\right)^{\gamma_{s}}$

4) Compute the parameter estimate $\hat{x}=\sum_{i=1}^{n} w^{S, i} \mathbf{x}^{S, i}$.

TABLE I

IMPORTANCE SAMPLING WITH BLIND PROGRESSIVE CORRECTION. 

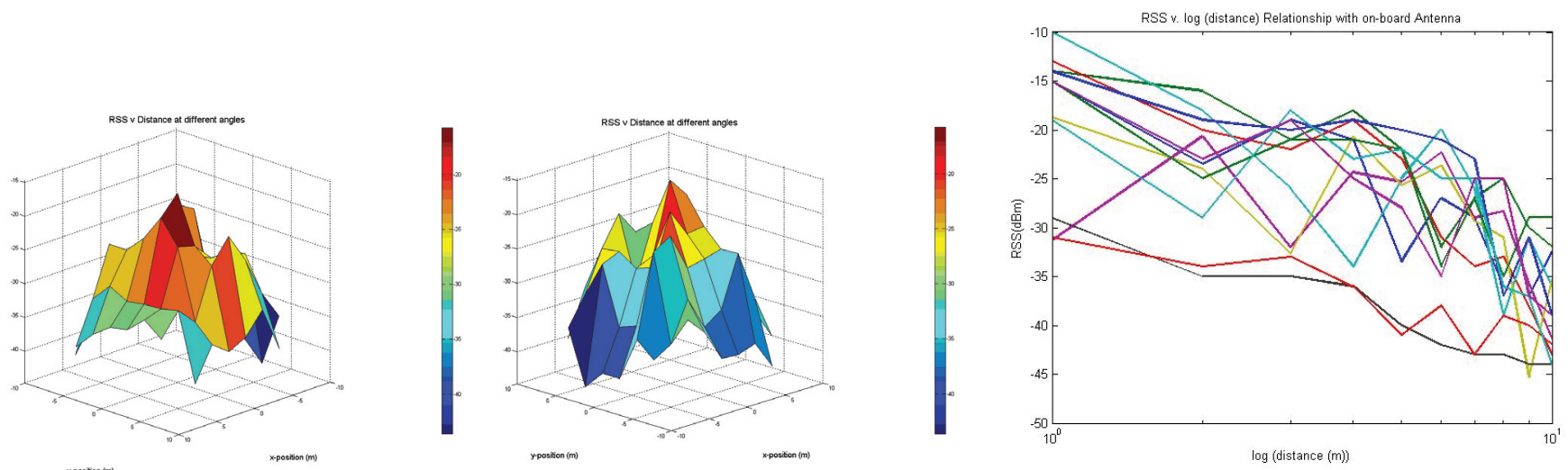

Fig. 1. RSS surface plot for chip antenna.

Fig. 2. RSS surface plot for chip antenna $\left(\right.$ rotated $\left.180^{\circ}\right)$.
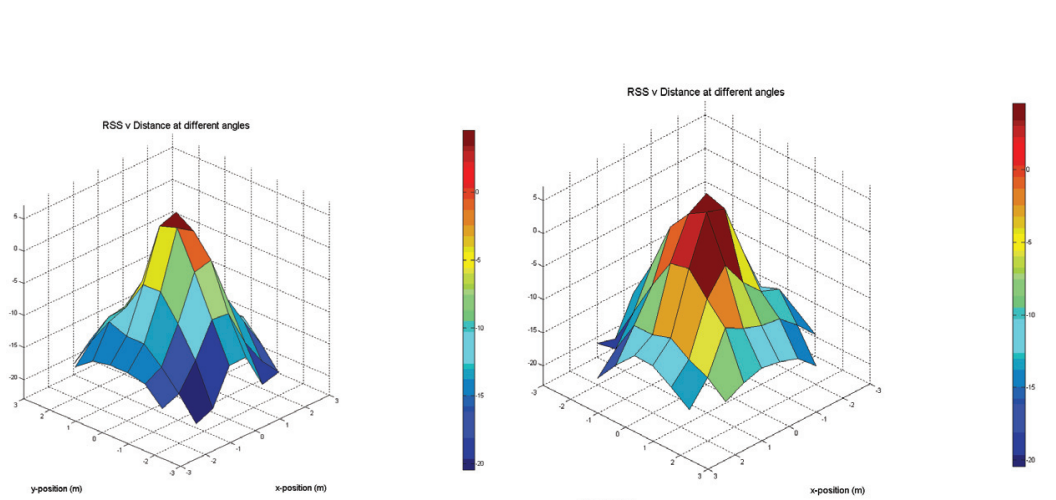

Fig. 4. RSS surface plot for monopol antenna. Fig. 5. RSS surface plot for monopol antenna (rotated $180^{\circ}$ ).

Fig. 3. Radial RSS profile of chip antenna.

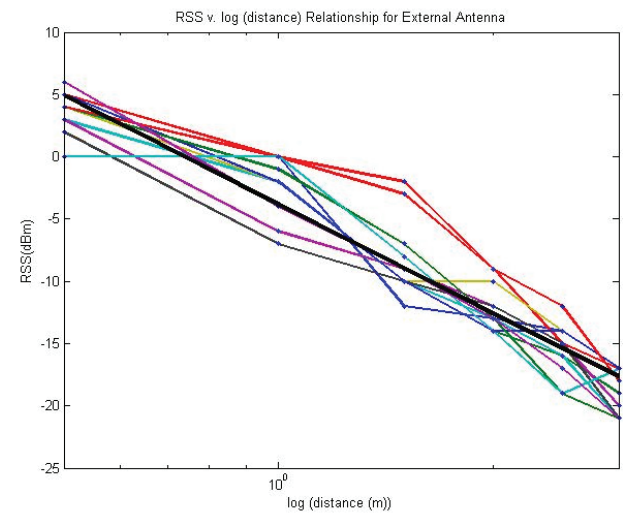

Fig. 6. Radial RSS profile of monopol antenna.

\section{A. Estimation of $P_{0, d B m}$ and $\alpha$}

A simple regression with current estimates $\hat{\mathbf{x}}$ at each stage $s$ can be done:

$$
\left[\begin{array}{c}
P_{0, d B m} \\
\alpha
\end{array}\right]=\left(F^{T} F\right)^{-1} F^{T} \mathbf{d}
$$

with $F$ being a $T \times 2$ matrix with the first column being 1 's and the second column having elements $-10 \log _{10}\left(r_{\nu(t)} / R_{0}\right)$ where $r_{\nu(t)}$ is the distance between communicating nodes of the $t$ th measurement. At the early stages, this might give bad estimates and it may also be problematic if there are huge outliers, in which case it maybe beneficial to remove the worst fitting measurements. A simple adaptive update maybe used to adjust the "ideal" antenna parameters.

\section{EXPERIMENTAL RESULTS}

Several localization experiments have been conducted. As we only have a small number of Imote 2 nodes, we conducted two "pseudo-network" experiments with four known transmitter locations and several unknown receiver locations. In these pseudo-network experiments, only one transmitter and one receiver were sequentially used to acquire the pairwise measurements at different locations (see Figure 7). All the pseudo-network experiments included only measurements between known and unknown nodes. No measurements between unknown nodes were made in pseudo-networks. However, for the real network experiments, measurements between unknown nodes were allowed.

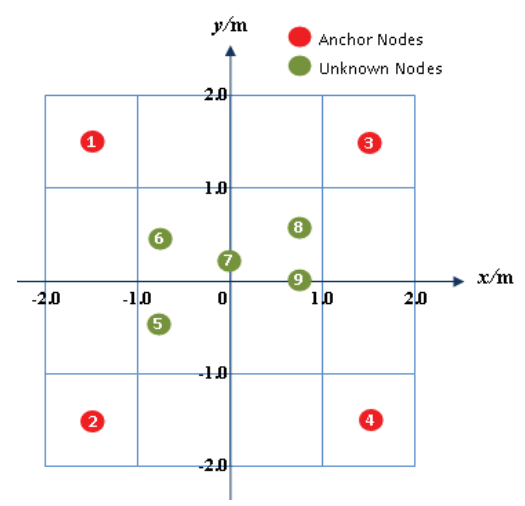

Fig. 7. Network setup.

\section{A. Pseudo-Networks}

The first experiment in a very small $1 \mathrm{~m}$ by $1.6 \mathrm{~m}$ surveillance area yielded $P_{0}=1.14 \mathrm{dBm}$ and $\alpha=0.45$ (see Figure 8). 
Location estimates are given in Figure 11. Due to the short distances and the rough quantization, these values are insensitive and transmit powers would have to be adjusted for a useful localization. The next experiment was done in a larger $3 \mathrm{~m}$ by $3 \mathrm{~m}$ area and the results look better (see Figures 9 and $12, \alpha=2.64, P_{0}=-5.54 \mathrm{dBm}$ ), in particular when the worst node was removed (Figures 10 and 13) which gives surprisingly close parameter estimates $\alpha=2.96, P_{0}=-3.96 \mathrm{dBm}$. In the last two cases the average RMS position estimation errors were $45 \mathrm{~cm}$ and $28 \mathrm{~cm}$.

\section{B. Real Networks}

These experiments use a similar setup in a $1.6 \mathrm{~m}$ by $2.5 \mathrm{~m}$ area on a conference table, at least $1 \mathrm{~m}$ away from any wall, but with 4 anchors and 2 unknown nodes. A central node is used to schedule other node pairs to make and report measurements to the central node.

Two issues were encountered in this scenario when measurements were transmitted using TCP/IP. Sending commands too quickly resulted in hanging TCP/IP packets and secondly the packet drop rate of large TCP/IP packets increased quickly, as a TCP/IP packet will be dropped if any of its smaller ZigBee fragments collides. Slowing down the central node by having a sleep period between individual commands, prevented TCP/IP locks. However, it was observed that certain pairs seemed to have a less omnidirectional behavior with reduced communications (see Table II). Comparison with the simulated measurements in Table II for the given "ideal" antenna model shows that nodes 3 and 4 are heavily biased. When performing simulation results, most often it is assumed that measurement noise is only a fraction of the distance. Clearly, the RSS measurements made with real hardware shows far greater and non-homogeneous deviations. For example, measurements between nodes 2 and 4 with $-30 \mathrm{dBm}$ and $-32 \mathrm{dBm}$ (correct value corresponding to ideal antenna model would be $-17.5 \mathrm{dBm}$ ) corresponds to distances of $796 \mathrm{~cm}$ and $932 \mathrm{~cm}$, while the true distance is $297 \mathrm{~cm}$, which is about 300 percent noise in distance. And between nodes 3 and 4, measurements are $1 \mathrm{dBm}$ and $3 \mathrm{dBm}$, corresponding to $68 \mathrm{~cm}$ and $58 \mathrm{~cm}$ (true: $9.7 \mathrm{dBm}, 160 \mathrm{~cm})$. Achieving only $20 \mathrm{~cm}$ RMSE localization error $\left(19 \mathrm{~cm}\right.$ with some adaptation of $P_{0, d B}$ and $\alpha$ ) from only 30 fairly noisy measurements, indicates a remarkable robustness of solving the Bayesian joint estimation problem by a PF with progressive correction. Moreover, a huge number of particles are avoided (approximately 4000 particles but with some modifications [9] as little as 200 maybe used).

The ideal way to acquire measurements would be to keep an internal array in the MAC layer where for every neighbor the RSS is filtered by an autoregressive moving averaging (ARMA) filter and stored with a system call to query its value. As our nodes run Linux, this involves kernel-user mode communications, which is more demanding to implement. For flexibility reasons, user process communication over sockets was preferred, and therefore either TCP/IP or UDP sockets had to be used. Preferably, connection-less UDP sockets should be used but due to time constraints and a possible bug in the UDP

\begin{tabular}{||c|rrrrrr||}
\hline \hline node & 1 & 2 & 3 & 4 & 5 & 6 \\
\hline \hline 1 & - & -14 & -17 & $\mathbf{- 7}$ & -9 & 1 \\
\hline 2 & -11 & - & $\mathbf{- 2 4}$ & $\mathbf{- 3 0}$ & -8 & -4 \\
\hline 3 & -15 & $\mathbf{- 2 3}$ & - & $\mathbf{3}$ & $\mathbf{0}$ & -10 \\
\hline 4 & $\mathbf{- 8}$ & $\mathbf{- 3 2}$ & $\mathbf{1}$ & - & -3 & -10 \\
\hline 5 & -8 & -9 & -2 & -5 & - & $\mathbf{- 8}$ \\
\hline 6 & $\mathbf{5}$ & -2 & -8 & $\mathbf{- 8}$ & -6 & - \\
\hline \hline
\end{tabular}

TABLE II

RSS MEASUREMENTS PER DBM IN REAL NETWORK. BOLD NUMBERS DEVIATE MORE THAN 5DBM FROM SIMULATED MEASUREMENTS.

\begin{tabular}{||c|rrrrrr||}
\hline \hline node & 1 & 2 & 3 & 4 & 5 & 6 \\
\hline \hline 1 & - & -9.7 & -17.5 & -15.8 & -10.8 & -2.2 \\
\hline 2 & -9.7 & - & -15.4 & -17.5 & -10.8 & -6.0 \\
\hline 3 & -17.5 & -15.4 & - & -9.7 & -6.5 & -13.2 \\
\hline 4 & -15.4 & -17.5 & -9.7 & - & -6.5 & -12.2 \\
\hline 5 & -10.8 & -10.8 & -6.5 & -6.5 & - & -2.7 \\
\hline 6 & -2.2 & -2.2 & -6.0 & -13.2 & -2.7 & - \\
\hline \hline
\end{tabular}

TABLE III

SIMULATED RSS MEASUREMENTS PER DBM FOR REAL NETWORK WITH $P_{0, d B m}=-3.91$ AND $\alpha=2.97$.

stack, TCP/IP was used for sending the RSS values returned by the radio driver in the network stack of the Linux kernel.

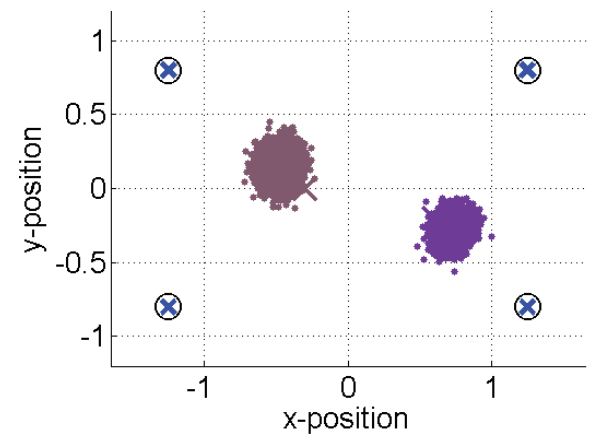

Fig. 14. Estimated locations for real network, $1.6 \mathrm{~m}$ by $2.5 \mathrm{~m}$ surveillance area, 2 unknown nodes. Estimates are ok (RMSE $21 \mathrm{~cm}$ ) despite some gross outliers mainly from two anchor nodes.

\section{CONCLUSION AND FUTURE WORK}

A centralized ranged-based self-localization algorithm based on particle filtering with progressive correction is proposed. The algorithm calculates distances by estimating the send power and loss factor of a simple log-normal shadowing model based on measured RSS values. A sensor network testbed consisting of 6 Crossbow Imote 2 sensors with 4 known and 2 unknown sensors positions, showed accurate localization with less than $16 \%$ error in a $3 \mathrm{~m} \times 3 \mathrm{~m}$ area, using less than 30 RSS measurements. For this simple model, an omnidirectional 


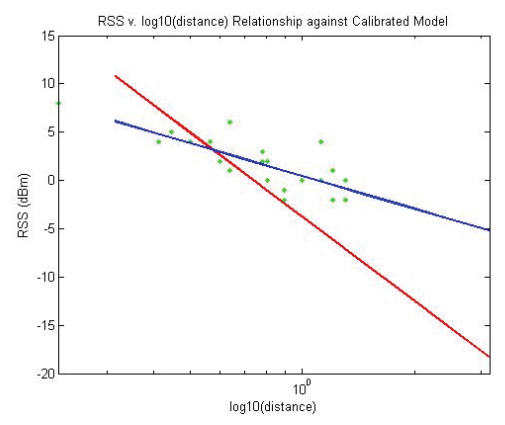

Fig. 8. Regression of RSS v $\log _{10}$ (distance) for a pseudo-network in a $1 \mathrm{~m} \times 1.6 \mathrm{~m}$ area.

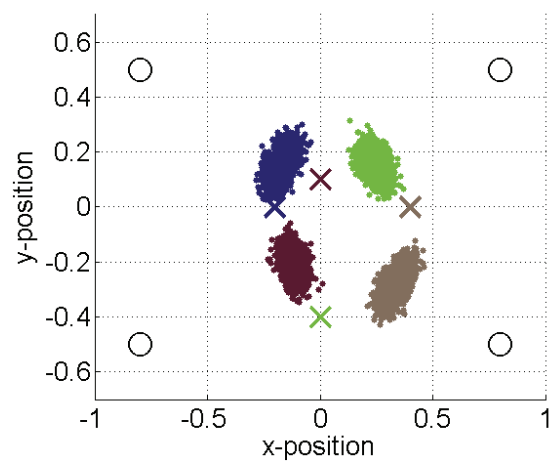

Fig. 11. Estimated locations for a pseudonetwork in a $1 \mathrm{~m} \times 1.6 \mathrm{~m}$ area. Estimates are bad as clusters get mixed up due to coarse quantization of RSS in this range.

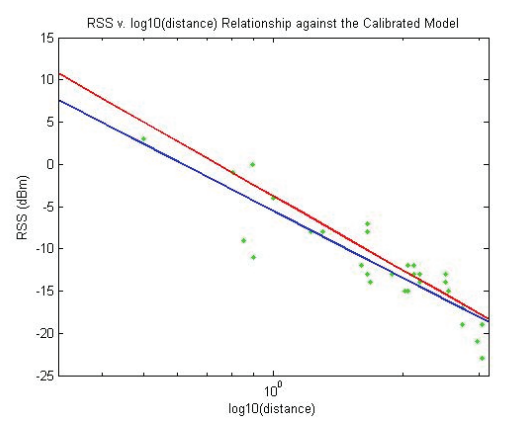

Fig. 9. Regression of RSS v $\log _{10}$ (distance) for a pseudo-network in a $3 \mathrm{~m} \times 3 \mathrm{~m}$ with 5 unknown nodes.

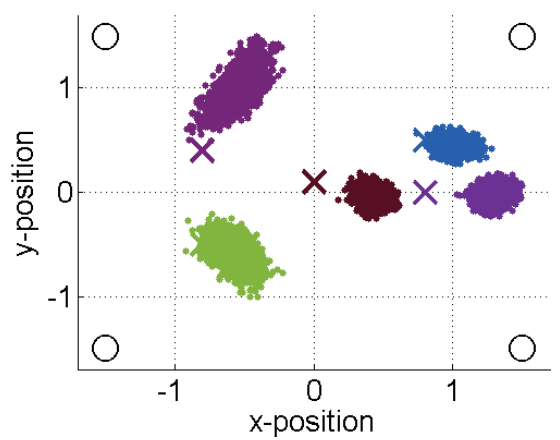

Fig. 12. Estimated locations for a pseudonetwork in a $3 \mathrm{~m} \times 3 \mathrm{~m}$ area with 5 unknown nodes. Estimates are ok (RMSE $45 \mathrm{~cm}$ ) but get biased by mainly one inaccurate sensor location.

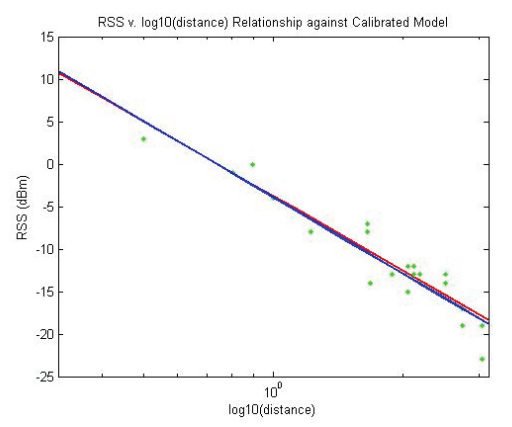

Fig. 10. Regression of RSS v $\log _{10}$ (distance) for a pseudo-network in a $3 \mathrm{~m} \times 3 \mathrm{~m}$ with 4 unknown nodes (outlier removed).

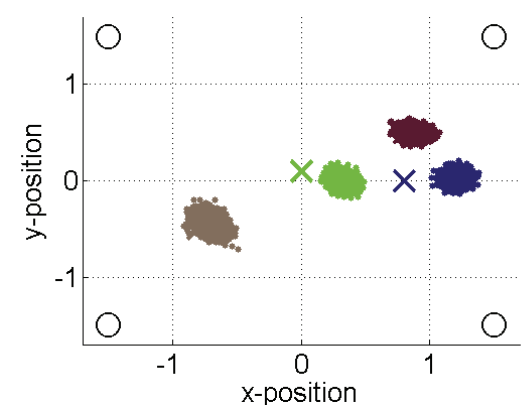

Fig. 13. Estimated locations for a pseudonetwork in a $3 \mathrm{~m} \times 3 \mathrm{~m}$ area with 4 unknown nodes (outlier removed). Estimates improve to RMSE of $27 \mathrm{~cm}$. external antenna had to be used as the on-chip antenna showed strong dipole characteristics, preventing reliable localization.

Future work includes a distributed and online version of the localization algorithm. The use of a simple ARMA filter may even improve robustness in RSS measurements for localization, however, at the cost of a reduced maximum tracking speed of moving nodes. An adaptive weighting between past and current measurement may reduce this effect. Another improvement may be to use individual measurement model parameters per node to better model environmental influences. To reduce the number of estimated parameters, individual send power strength may then have to be transmitted as well. The current ad hoc regression for the measurement model parameters may be integrated into the PF by RaoBlackwellization.

\section{REFERENCES}

[1] A. Baggio and K. Langendoen, "Monte Carlo localization for mobile wireless sensor networks," Ad Hoc Networks, vol. 6, no. 5, Jul. 2008.

[2] M. Brazil, M. Morelande, B. Moran, and D. Thomas, "Distributed self-localisation in sensor networks using RIPS measurements," in International Conference on Wireless Networks, 2007.

[3] B. Dil, S. Dulman, and P. Havinga, "Range-based localization in mobile sensor networks," in Wireless Sensor Networks, ser. LNCS, vol. 3868. Springer Berlin, 2006, pp. 164-179.

[4] E. Elnahrawy, X. Li, and R. P. Martin, "The limits of localization using signal strength: a comparative study," in IEEE SECON 2004, October 2004, pp. $406-414$.
[5] L. Hu and D. Evans, "Localization for mobile sensor networks," in MobiCom '04. New York, NY, USA: ACM, 2004, pp. 45-57.

[6] R. Huang and G. V. Záruba, "Location tracking in mobile ad hoc networks using particle filters," Journal of Discrete Algorithms, vol. 5, no. 3, pp. $455-470,2007$.

[7] D. Lymberopoulos, Q. Lindsey, and A. Savvides, "An Empirical Characterization of Radio Signal Strength Variability in 3-D IEEE 802.15.4 Networks Using Monopole Antennas," in Wireless Sensor Networks, ser. LNCS, vol. 3868. Springer-Verlag, 2006, pp. 326-341.

[8] G. Mao, B. Fidan, and B. D. Anderson, "Wireless sensor network localization techniques," Computer Networks, vol. 51, no. 10, pp. 2529 $-2553,2007$.

[9] M. R. Morelande, B. Moran, and M. Brazil, "Bayesian node localization in wireless sensor networks," in ICASSP 2008, 2008, pp. 2545-2548.

[10] Morelli, C. and Nicoli, M. and Rampa, V. and Spagnolini, U. and Alippi, C., "Particle filters for RSS-based localization in wireless sensor networks: An experimental study," in ICASSP 2006, vol. 4, 2006, pp. 957-960.

[11] R. L. Moses, D. Krishnamurthy, and R. Patterson, "A self-localization method for wireless sensor networks," EURASIP Journal of Applied Signal Processing, vol. 4, pp. 348-358, 2003.

[12] C. Musso, N. Oudjane, and F. L. Gland, "Improving regularised particle filters," in Sequential Monte Carlo Methods in Practice, A. Doucet, N. de Freitas, and N. Gordon, Eds. Springer-Verlag, 2001.

[13] O. Ozdemir, R. Niu, and P. K. Varshney, "Tracking in wireless sensor networks using particle filtering: physical layer considerations," IEEE Trans. Signal Processing, vol. 57, no. 5, pp. 1987-1999, May 2009.

[14] N. Patwari, I. Hero, A.O., M. Perkins, N. Correal, and R. O'Dea, "Relative location estimation in wireless sensor networks," IEEE Trans. Signal Processing, vol. 51, no. 8, pp. 2137 - 2148, Aug. 2003.

[15] B. Silverman, Density Estimation for Statistics and Data Analysis. Chapman and Hall, 1986. 